DOI: 10.12957/demetra.2015.16464

\title{
Doação de leite materno ao banco de leite humano: conhecendo a doadora
}

\section{Donation of breastmilk to human milk bank: knowing the donor}

\author{
Emily Semenov Silva' \\ Laurita Ester de Jesus² \\ Emanuele Batistela dos Santos' \\ Nely Alves de Castro ${ }^{3}$ \\ Lorena Barbosa Fonsecal \\ 1 Universidade Federal de Mato Grosso, \\ Faculdade de Nutrição, Departamento de \\ Alimentos e Nutrição. Cuiabá-MT, Brasil. \\ ${ }^{2}$ Universidade Federal de Mato Grosso, Instituto \\ de Ciências Humanas e Sociais, Departamento de \\ Serviço Social. Cuiabá-MT, Brasil. \\ ${ }^{3}$ Universidade Federal de Mato Grosso, Hospital \\ Universitário Júlio Müller. Cuiabá-MT, Brasil. \\ Corrrespondência / Correspondence \\ Lorena Barbosa Fonseca \\ E-mail: Iorenanutricao@gmail.com
}

\section{Resumo}

Objetivo: Descrever o perfil das doadoras de leite humano ao Banco de Leite Humano de um hospital universitário do município de Cuiabá-MT. Método: Estudo observacional, de corte transversal realizado com todas as nutrizes cadastradas no Banco de Leite Humano como doadoras externas, utilizando questionário semiestruturado entregues às nutrizes durante a coleta domiciliar de leite e, para as ex-doadoras aplicados, via telefone, contendo variáveis sociodemográficas, pré-natal e processo de doação. Resultados e Discussão: Participaram 70 doadoras com idade média de 28,5 anos, $77,14 \%$ eram casadas ou em união estável, $36,76 \%$ tinham ensino superior completo e $66,67 \%$ trabalhavam. Não houve relação entre a idade e a doação. O altruísmo foi a principal razão pelas mulheres procurarem o Banco de Leite Humano. A doação pode ser justificada pelo instinto materno e o sentimento de solidariedade. O início da doação se deu um mês após o nascimento do filho, sendo um período de grande procura ao Banco de Leite e uma boa oportunidade para o estímulo à amamentação; 88,24\% relataram não ter tido dificuldades para iniciar a doação e o período médio de doação foi de 90 dias. Conclusão: Os dados apontam a importância da educação em saúde ainda no pré-natal, pois estão sensíveis ao aprendizado. Ressalta-se a necessidade de os bancos de leite humano conhecerem o perfil das doadoras para alcançar a abordagem de divulgação adequada e a captação de novas doações.

Palavras-chave: Bancos de Leite. Leite Humano. Aleitamento Materno. 


\section{Abstract}

Objective: To analyze the profile of human milk donor of a Human Milk Bank of a university hospital in the city of Cuiabá-MT, Brazil. Methods: observational, cross-sectional study conducted with all nursing mothers enrolled in the human milk bank as external donor using semi-structured questionnaire administered during the home collection of milk and, to the former donor, administered via telephone calls. We collected information concerning social demographic variables, prenatal care and donation process. Results and discussion: 70 donors participated and the average age was 28.5 years old; $77.14 \%$ were married or in stable union, $36.76 \%$ had completed higher education and $66.67 \%$ were active in work. There was no relation between the age of donor and donation. Altruism was the main reason for women seeking the human milk bank. Donation can be justified by the maternal instinct and by a sense of solidarity. The beginning of the donation took place a month after the birth of child, being a period of great demand to human milk bank and a good opportunity for the stimulus to breastfeeding; $88.24 \%$ reported no difficulties to start the donation and the median donation period was 90 days. Conclusion: data indicated the importance of health education in prenatal care, because the mothers are more sensitive to learning in this period. We highlight the need of the human milk banks to know the profile of donors in order to use the proper advertising approach and get new donations.

Key words: Milk Banks. Human Milk. Breast Feeding.

\section{Introdução}

O leite materno (LM) é o melhor alimento para a criança, devido a sua importância nutricional, presença de fatores imunológicos e contribuição para o fortalecimento do vínculo entre mãe e filho. A recomendação atual da Organização Mundial da Saúde é que ele seja oferecido de forma exclusiva até os seis meses de vida, e complementado até pelo menos dois anos de idade. ${ }^{1}$

A partir de 1985, houve expansão dos Bancos de Leite Humano (BLH) no Brasil e a criação em 1998 da Rede Brasileira de Bancos de Leite Humano. Os BLH desempenham papel importante nas ações de promoção, proteção e apoio ao aleitamento materno (AM), bem como oferta de leite humano ordenhado pasteurizado, prioritariamente aos recém-nascidos prematuros e/ou de baixo- 
peso, que não sugam, infectados, dentre outros. ${ }^{2}$ Os BLH constituem, desta forma, alternativa segura e importante para manutenção do consumo de leite humano nos casos em que a criança não pode ser amamentada diretamente ao seio de sua mãe.

Cabe ressaltar que o leite humano é oriundo de doações voluntárias de nutrizes que possuem produção excedente de leite e que estejam amamentando seus filhos.

Apesar da ampla divulgação nos meios de comunicação de massa e serviços de saúde sobre a importância da doação de leite materno, dados do Ministério da Saúde demonstraram que em 2013 e 2014 houve redução de aproximadamente 11\% e 8\%, respectivamente, no número de doadoras em comparação ao ano de 2012 no país. ${ }^{3,4}$ Neste sentido, investigações conduzidas com o intuito de conhecer o perfil das nutrizes doadoras de leite humano (LH) são relevantes, considerando seu potencial para subsidiar o desenvolvimento de estratégias eficazes para captação de novas doadoras.

A literatura aponta que as doadoras, em sua maioria, são adultas jovens, casadas ou em união estável, e possuem alto nível de escolaridade. Em geral são primíparas e realizaram parto cesárea. Com relação às razões que levam à doação de leite, o altruísmo aparece como a principal, mas razões como prevenção do ingurgitamento mamário também foram citadas por algumas mães. ${ }^{5-10}$ Deste modo, este estudo teve como objetivo descrever o perfil das doadoras de LH ao Banco de Leite Humano de um hospital universitário do município de Cuiabá-MT.

\section{Metodologia}

Trata-se de estudo observacional, de corte transversal, desenvolvido no Banco de Leite Humano de um hospital de Cuiabá-MT, como um dos produtos do Programa de Educação pelo Trabalho para a Saúde (PRÓ/PET-Saúde) da Universidade Federal de Mato Grosso. Foram incluídas no estudo todas as nutrizes cadastradas no BLH como doadoras externas, de janeiro de 2013 a novembro de 2014. Foram excluídas as mulheres que realizavam a ordenha de leite no BLH para seu próprio filho (doadoras internas) e mulheres cadastradas sem episódio de doação após janeiro de 2013.

Os dados foram coletados por meio de questionário semiestruturado elaborado pelas próprias pesquisadoras. Os questionários foram entregues às nutrizes pela equipe do BLH durante a coleta domiciliar de leite. Para as ex-doadoras, os questionários foram aplicados via telefone. Todas as doadoras foram contatadas previamente via telefone por monitores do PET-Saúde, recebendo explicações sobre a pesquisa, orientações para preenchimento do questionário e para a assinatura do Termo de Consentimento Livre e Esclarecido, elaborado de acordo com a Resolução n 466/2012 do Conselho Nacional de Saúde. Foram coletadas informações sociodemográficas, sobre o prénatal e processo de doação de leite. 
Para tabulação e análise dos dados, foi utilizado o software Epi Info versão 7. As informações quantitativas foram analisadas de forma descritiva, por meio de medidas de frequência (relativa e absoluta) e de tendência central. Para o estudo qualitativo, as variáveis foram analisadas de acordo com os procedimentos da análise de conteúdo categorial. As respostas registradas pelas próprias doadoras foram analisadas e categorizadas a partir de seu conteúdo, por dois pesquisadores, com o objetivo de garantir concordância igual ou superior a 70\% em relação à identificação e nomeação das categorias. ${ }^{11}$

Este estudo foi aprovado pelo Comitê de Ética do Hospital Universitário Júlio Müller, sob parecer n⿳0 735.627, de 31 de julho de 2014.

\section{Resultados e Discussão}

Participaram do estudo 70 doadoras de LH, com idade média de 28,5 anos. A maioria ( $\mathrm{n}=54$; $77,14 \%)$ era casada ou vivia em união estável; 36,76\% ( $n=25)$ tinham ensino superior completo e $66,67 \%$ trabalhavam fora de casa $(n=46)$. A renda familiar mediana foi de $\mathrm{R} \$ 2.000,00$, variando de $\mathrm{R} \$ 700,00$ a $\mathrm{R} \$ 20.000,00(\mathrm{p} 25=\mathrm{R} \$ 1.000,00 ; \mathrm{p} 75=\mathrm{R} \$ 4.000,00)$.

No estudo de Dias et al, ${ }^{6}$ realizado em um BLH de um hospital universitário, a maioria das doadoras eram adultas jovens, assim como no presente estudo, que apresentou uma média de idade de 28,5 anos. Entretanto, segundo Fonseca-Machado et al., ${ }^{12}$ parece não haver relação entre a idade materna e a prática da doação de leite.

Em relação ao estado civil, resultados semelhantes foram encontrados por Galvão et al. ${ }^{7}$ e Fonseca-Machado et al., ${ }^{12}$ apontando que a maioria das doadoras eram casadas. Acredita-se que a presença e apoio do parceiro encorajam a mulher tanto a amamentar quanto a doar seu leite. ${ }^{13}$ Quanto à escolaridade, estudos mostram que mulheres com maior nível de formação tendem a amamentar por mais tempo, possivelmente porque o grau de instrução interfere na assimilação das orientações recebidas sobre a prática do aleitamento materno e também na decisão quanto à doação de LH.9.14,15

Quanto à ocupação, pesquisas mostram o elevado número de doadoras que possuem trabalho remunerado, corroborando o resultado deste estudo. Isto pode ser explicado, pelo menos em parte, pelo crescimento da participação da mulher no mercado de trabalho. Por outro lado, este fator também se associa à introdução de alimentos complementares precocemente, o que contribui para a redução da produção do LM e, consequentemente, da possibilidade de sua doação. ${ }^{6,8,15}$

Entre os anos 1940 e 1970, as doadoras eram em sua maioria pobres e, muitas vezes, comercializavam seu leite para aumentar o rendimento familiar. Com a proibição da comercialização 
do LH, a doação passou a ser estritamente voluntária. ${ }^{16}$ Nesta pesquisa, a renda mediana das doadoras situou-se no correspondente a 2,5 salários mínimos (SM) vigentes na época do estudo. Estudo de Prado ${ }^{14}$ identificou que a maioria das doadoras tinha renda familiar de aproximadamente três SM. Vale destacar que, diferentemente dos registros dos primeiros BLH, nos quais não havia nutrizes pertencentes às camadas sociais mais elevadas, ${ }^{17}$ nesta pesquisa observou-se que $25 \%$ das doadoras apresentavam renda familiar entre $\mathrm{R} \$ 4.000,00$ e $\mathrm{R} \$ 20.000,00$.

Em relação às características gestacionais, o tipo de parto mais frequente foi o cesáreo $(n=44$; $62,86 \%)$ e o pré-natal foi realizado predominantemente na rede privada de saúde $(\mathrm{n}=40 ; 57,14 \%)$. Com relação ao número de filhos, das 57 respostas obtidas, a maioria das doadoras (n=41; 71,93\%) era primípara, corroborando outros estudos. Em geral, quando a mulher está amamentando seu primeiro filho, sente-se mais insegura e tende a procurar com mais frequência os serviços prestados pelo BLH. ${ }^{9,14,18}$

Observou-se, neste estudo, uma média de dez consultas realizadas durante o pré-natal, estando, assim como em outras pesquisas, ${ }^{8,11}$ acima do preconizado pelo Ministério da Saúde que é de, no mínimo, seis consultas..$^{19}$ Considera-se alto o número de mães que relataram não ter recebido qualquer orientação sobre aleitamento materno durante as consultas de pré-natal $(\mathrm{n}=32 ; 46,38 \%)$. Porém, a maioria $(\mathrm{n}=37 ; 53,62 \%)$ referiu ter recebido algum tipo de orientação sobre aleitamento materno durante esse período. De fato, o acompanhamento pré-natal favorece o vínculo entre a gestante e o profissional de saúde, e por isso é considerado um ótimo momento para a realização de orientações sobre aleitamento materno e doação de leite. ${ }^{20,21}$ Segundo Dias, ${ }^{6}$ é frequente observar que as mães recebem informações sobre amamentação apenas pouco antes do nascimento do filho, dificultando a posterior lactação. Orientações durante toda a gestação são importantes para garantir sensibilização da mãe e contribuem tanto para o aleitamento materno quanto para a doação do leite excedente.

No presente estudo, em relação à fonte de informação sobre como realizar a doação de leite humano e sobre o serviço prestado pelo BLH, em primeiro lugar, apareceram a família e amigos ( $\mathrm{n}=27 ; 38,57 \%)$, seguidos dos serviços de saúde $(\mathrm{n}=22 ; 31,43 \%)$, demonstrando a influência da comunidade, representada por pessoas íntimas, na conduta adotada pela gestante e nutriz, bem como das orientações prestadas pelos serviços de saúde desde a primeira consulta de pré-natal até o parto. ${ }^{9}$ Os meios de comunicação apareceram em última posição ( $\left.n=15 ; 21,43 \%\right)$, o que reflete a pequena utilização deste meio de comunicação como marketing social para o estímulo à doação de leite. De modo oposto, no estudo de Pinto, ${ }^{10}$ as doadoras relataram ter conhecimento sobre a necessidade da doação, principalmente pela televisão. Considerando sua capacidade para atingir um grande público em um curto espaço de tempo, por se tratar de um meio de comunicação em massa, acredita-se que os meios de comunicação poderiam ser mais utilizados para este fim pelo BLH estudado neste trabalho. 
Quanto às razões que levaram as mães a procurarem o BLH, a mais relatada foi saber que o banco precisava de doações $(n=27 ; 39,71 \%)$, isto é, apresentava demanda por parte de mães e crianças que por alguma razão não estavam realizando aleitamento materno ao seio. Este resultado foi semelhante ao encontrado por outros estudos, que sugerem o altruísmo - ação que beneficia outro indivíduo, solidariedade, ato voluntário, não remunerado - como uma das principais razões apontadas para a doação de LH. ${ }^{9,11}$ Segundo Silva, ${ }^{22}$ a doação de leite se justifica, em alguns casos, pela identificação com o sentimento da mãe receptora. A segunda razão mais relatada foi o excesso de produção láctea $(n=23 ; 33,82 \%)$, que pode provocar o ingurgitamento mamário. Em alguns estudos, esta superou o altruísmo dentre as razões que levavam à doação. ${ }^{9}$

O início da doação ocorreu aproximadamente um mês após o nascimento do filho (mediana $=30$ dias; $\mathrm{p} 25=11 ; \mathrm{p} 75=60)$. Acredita-se que o primeiro mês é um período em que ocorre maior procura aos serviços de saúde pela nutriz, sendo assimuma oportunidade para o BLH realizar estímulo para a amamentação e doação de leite. ${ }^{13} \mathrm{~A}$ maioria $(\mathrm{n}=60 ; 88,24 \%)$ relatou não ter tido dificuldades para iniciar a doação. Para aquelas que referiram algum obstáculo, estes foram associados à dificuldade na ordenha do leite, à mama ingurgitada e ao fato de as nutrizes considerarem não possuir quantidade de leite suficiente para doar. No estudo de Neves et al., ${ }^{23}$ boa parte referiu não ter problemas para iniciar a doação, mas para aquelas que citaram haver algum, as dificuldades citadas foram a falta de leite, a preguiça, o medo, a falta de tempo, doenças graves, entre outros.

Neste estudo, o período mediano de doação de leite foi de 90 dias (p25=30,5; p75=135). Considerando que neste mesmo estudo a doação teve início aproximadamente um mês após o nascimento do filho, acredita-se que a interrupção da doação nesse período está associada ao desmame precoce, caracterizado pela introdução da alimentação complementar, o que coincide com o retorno da nutriz ao trabalho. Sabe-se que o desmame causa a redução da produção láctea e, consequentemente, da doação do LH. ${ }^{6,11}$

Neste estudo, procurou-se ainda investigar a percepção das doadoras quanto à prática da doação de leite. As falas foram analisadas e agrupadas nas categorias "altruísmo", "papel cidadão", "prevenção de desperdício", "influência de alguém”, "conhecimento de que o BLH precisava", "produção láctea excedente", "alívio da dor nos seios", "impossibilidade anterior de amamentar", "não ter sido amamentada", "conhecimento da importância do leite materno" e "conhecimento de que o LH doado não é comercializado". 
Pode-se perceber que de fato, assim como identificado no estudo quantitativo, a categoria "altruísmo" foi a mais citada, mencionada por 19 doadoras, como pode ser observado nos relatos abaixo:

Eu me sinto muito bem ao doar leite, penso que assim como outros bebês precisam minha filha poderia estar em situação igual [...]. (D14)

Sinto muito feliz em saber que estou contribuindo para salvar vidas de um bebê prematuro em uma UTI e realizar o sonho da maternidade [...] É um sentimento singular de amor ao próximo [...]. (D22)

[...] e poder estar ajudando outras crianças além da minha com o leite humano é algo que não tem preço [...]. Há mais felicidade em dar do que há em receber. (D11)

Sinto que estou contribuindo para uma vida saldável de alguns bebês que precisam. Doar leite é doar amor, gotinhas de saúde. (D2)

Outro motivo observado nos relatos das doadoras foi o "papel cidadão", mencionado por 15 nutrizes:

[...] Sinto orgulho de ver que meu leite pode alimentar não só a minha filha. Me sinto feliz, me sinto bem, com saúde [...]. (D15)

Me sinto cumprindo o dever de uma cidadã. Faço a minha parte. (D8)

A preocupação com o desperdício de leite materno foi citada por três participantes, incluídas na categoria "prevenção de desperdício":

[...] Antes de doar leite eu jogava muito leite fora, agora tenho pra quem doar. Pra mim é um privilégio doar e enquanto eu tiver leite suficiente vou doar. Pra mim leite é vida e vida pra mim é tudo. (D14)

[...] Porque às vezes fico com os seios doendo de tão cheio, e agora como estou doando não cinto mas e também penso nas crianças que precisa, em vez de desperdiçar melhor ajudar elas. (D10)

[...] Uma coisa tão simples. Não leva nem 15 min pra que possa encher um potinho. Muitas vezes muitas outras mães pudessem doar pois é o leite que vaza do peito [...]. (D21).

Outras categorias, como "influência de alguém", "conhecimento de que o BLH precisava", "produção láctea excedente", "alívio da dor nos seios", "impossibilidade anterior de amamentar", 
"não ter sido amamentada", "conhecimento da importância do leite materno" e "conhecimento de que o LH doado não é comercializado" foram relatadas, cada uma, por apenas uma doadora.

Para conhecer as razões que levaram ao término da doação, realizou-se entrevista apenas com as ex-doadoras. Os relatos foram agrupados nas categorias "redução da produção láctea", "retorno às rotinas cotidianas", doenças/medicação", "mudança de cidade", "perda de contato com o BLH", "falecimento de cônjuge", "falta de apoio familiar", "intenção de parar de amamentar", "perda de peso do filho" e "perda do hábito de ordenha”. As categorias "redução da produção láctea” (n=20) e "retorno ao trabalho/estudo" $(\mathrm{n}=13)$ foram as mais citadas:

Ele começou a comer outros alimentos e com isso diminuiu a suç̧ão e produção do leite materno. (E41)

Não produzia muito leite mais e o bebê mamava tudo. (E52)

Foi diminuindo a produção. O bebê mamou pouco e faltou estímulo para a produção. (E69)

Voltei a estudar e retirava só para deixar para meu bebê. (E39)

Após a licença maternidade retornei ao trabalho e não consegui adequar a rotina para a coleta da doação. (E58)

Voltei a trabalhar e não tinha mais tempo para estar ordenhando o leite e diminuiu a produção. (E59)

A categoria "doenças/medicação" apareceu em terceiro lugar, sendo citada por quatro participantes.

[...] fiz uma cirurgia e tive que usar medicamentos como antibióticos e com isso o leite secou. (E37)

[...] por motivos de saúde, tive cálculo renal, infecção intestinal e tomava uma medicação muito forte. Devido à medicação ser forte meu leite foi diminuindo. (E47)

Outras razões, incluídas nas categorias "mudança de cidade", "perda de contato com o BLH", "falecimento de cônjuge", "falta de apoio familiar", "intenção de parar de amamentar", "perda de peso do filho" e "perda do hábito de ordenha" também foram citadas.

Esses dados corroboram os encontrados por Alencar e Seidl ${ }^{11}$ e Fonseca-Machado et al. ${ }^{12}$ Destacase a importância da implementação de políticas públicas que favoreçam a promoção, proteção e apoio ao AM, como a ampliação do período de duração da licença-maternidade, que hoje no Brasil é de 120 dias, ${ }^{24}$ uma vez que este pode ter sido um dos fatores associados à interrupção das doações. 


\section{Conclusão}

Neste estudo, as doadoras de leite humano, que eram, na maioria, adultas jovens, casadas ou em união estável, com bom nível de escolaridade e trabalhadoras remuneradas, tiveram conhecimento do banco de leite humano através de família e amigos.

Os principais fatores que levaram à doação foram a demanda do BLH, a partir da necessidade das mães e crianças que dele dependiam e o excesso de produção láctea. Já em relação à interrupção, destacou-se o retorno às atividades de trabalho e estudo, além da redução da produção láctea. Os meios de comunicação apareceram em última posição, quando questionadas sobre a forma como tomaram conhecimento dos serviços prestados pelo BLH. Dessa forma, percebe-se que há necessidade de o BLH constituir programas apropriados para garantir captação de número adequado de doadoras, já que é sua responsabilidade, juntamente com o Posto de Coleta de Leite Humano, promover esse tipo de ação.

A doação de leite foi percebida pelas doadoras como uma ação de solidariedade essencial para salvar vidas de crianças impossibilitadas de serem amamentadas ao seio de sua mãe. É importante considerar, ainda, que o contexto social em que a mulher está inserida influencia sobremaneira em sua atitude em relação à doação. Assim, o apoio de pessoas próximas, como o cônjuge, e o suporte institucional agem positivamente para a decisão do ato de doar.

Os dados apontam a importância da educação em saúde, abordando o aleitamento materno e a doação de LH ainda no pré-natal, pois é nesse período que a mãe começa a se preparar para a lactação e está sensível ao aprendizado. Deve-se também expandir as orientações às puérperas, pois muitas vezes estas apresentam dificuldades em relação à amamentação e acabam interrompendo-a, reduzindo as chances de doação.

Considerando estas informações, ressalta-se a necessidade de os BLH conhecerem o perfil das doadoras para alcançar a abordagem de divulgação adequada e a captação de novas doações. E ainda, investigar as dificuldades relacionadas à interrupção da doação de LH, importante para subsidiar o desenvolvimento de intervenções mais direcionadas.

\section{Agradecimentos}

Ao Programa Nacional de Reorientação da Formação Profissional em Saúde (PRÓ-SAÚDE); Programa de Educação pelo Trabalho para a Saúde (PET-Saúde); Banco de Leite Humano do Hospital Universitário Júlio Müller; e doadoras do Banco de Leite Humano do Hospital Universitário Júlio Müller. 


\section{Referências}

1. Brasil. Ministério da Saúde. Saúde da criança: nutrição infantil aleitamento materno e alimentação complementar. Brasília: Ministério da Saúde; 2009.

2. Brasil. Agência Nacional de Vigilância Sanitária. Banco de leite humano: funcionamento, prevenção e controle de riscos. Brasília: Agência Nacional de Vigilância Sanitária; 2008.

3. Rede Brasileira de Bancos de Leite Humano [Internet]. Disponível em: http://producao.redeblh.icict. fiocruz.br/portal_blh/blh_brasil.php

4. Portal Brasil. Lançada campanha para incentivar doação de leite materno [Internet]. Brasília: 2014. Disponível em: URL: http://www.brasil.gov.br/saude/2014/05/lancada-campanha-para-incentivardoacao-de-leite-materno

5. Daroz SM. Análise do perfil das doadoras de leite materno do hospital fornecedores de cana, em Piracicaba. Anais $7^{a}$ Mostra Acadêmica UNIMEP; 2009; Santa Bárbara d’Oeste e Lins, Piracicaba. p. 1-5.

6. Dias RC, Baptista IC, Gazola S, rona MSS, Matioli G. Perfil das doadoras do banco de leite humano do Hospital Universitário de Maringá, Estado do Paraná, Brasil. Acta Sci. Health Sciences 2006; 28(2):153-158.

7. Galvão MTG, Vasconcelos SG, Paiva SS. Mulheres doadoras de leite humano. Acta Paulista de Enfermagem 2006; 9(2):157-61.

8. Lourenço D, Bardini G, Cunha L. Perfil das doadoras do banco de leite humano do Hospital Nossa Senhora da Conceição, Tubarão/SC. Arquivos Catarinenses de Medicina 2012; 41(1):22-27.

9. Santos DT, Oliveira Vannuchi MT, Benevenuto Oliveira MM, Dalmas JC. Perfil das doadoras de leite do banco de leite humano de um hospital universitário. Acta Scientiarum. Health Sciences 2009; 31(1):15-21.

10. Pinto MCLM, Campelo TC, Ramos VC, Lima MER. Alegações maternas para doação de leite humano ao banco de leite em Teresina-Piauí. Revista Interdisciplinar NOVAFAPI 2012; 5(2):5-2.

11. Alencar LCE, Seidl EMF. Doação de leite humano: experiência de mulheres doadoras. Revista Saúde Pública 2009; 43(1):70-77.

12. Fonseca-Machado MOF; Parreira BDM; Dias FA; Costa NS; Monteiro JCS; Gomes-Sponholz FG. Caracterização de nutrizes doadoras de um banco de leite humano. Ciência Cuidado e Saúde 2013; 12(3):529-538.

13. Silva PLN, Jorge JCT, Fonseca JR, Pereira ACA, Oliveira VGR. Perfil das mães doadoras de um banco de leite humano. Revista enfermagem UFPE [Internet] 2013; 7(7):4635-4640.

14. Prado MM. Banco de leite humano do município de Varginha-MG: Perfil das doadoras e causas de descarte do leite. [dissertação]. Lavras, MG: Universidade Federal de Lavras, Curso de Mestrado de Ciência dos Alimentos; 2009.

15. Genovez CB, Uchimura TT, Santana R, Nishida FS. Banco de leite humano: uma análise das diferenças entre doadoras adultas e adolescentes. Acta Scientiarum. Health Sciences 2011; 33(2): 211-218. 
16. Almeida JAG, Novak FR. Amamentação: um híbrido natureza-cultura. Jornal Pediatria 2004; 80(5):S119-S125.

17. Almeida JAG. Amamentação: um híbrido natureza cultura. 20 ed. Rio de Janeiro: FIOCRUZ; 1999.

18. Braga ASC, Andrade PC, Ferreira RAM, Davis JRP, Santos LC, Pereira SCL. Caracterização das mães doadoras e do leite doado ao banco de leite humano referência em Minas Gerais: subsídio para estratégias públicas de otimização do serviço. Anais XV Seminário Sobre a Economia Mineira; 2012; Diamantina, Belo Horizonte. p. 1-14.

19. Brasil. Ministério da Saúde. Manual técnico: pré-natal e puerpério. Atenção qualificada e humanizada. Brasília: Ministério da Saúde; 2005.

20. Landerdahl MC, Cabral FB, Ressel LB, Gonçalves MO, Martins FB. Percepção de mulheres sobre atenção pré-natal. Esc Anna Nery R. Enferm. 2007; 11(1):105-111.

21. Souza VB, Roecker S, Marcon SS. Ações educativas durante a assistência pré-natal: percepção de gestantes atendidas na rede básica de Maringá-PR. Revista Eletrônica de Enfermagem 2011; 13(2):199-210.

22. Silva SL. Alegações materna para doação de leite humano [dissertação]. Recife: Universidade Federal de Pernambuco, Pós Graduação em Saúde da Criança e do Adolescente; 2010.

23. Neves LS; As MVS; Matar, MJG; Galisa, MS. Doação de leite humano: dificuldades e fatores limitantes. O Mundo da Saúde 2011; 35(2):156-161.

24. Brasil. Decreto-Lei no 5.452, de 1 maio de 1943. Consolidação das Leis do Trabalho. Dário Oficial da União 9 ago. 1943; Seção 1:11937. 
